\title{
Correction to: Effectiveness and safety of nivolumab in patients with head and neck cancer in Japanese real-world clinical practice: a multicentre retrospective clinical study
}

\author{
Nobuhiro Hanai ${ }^{1} \cdot$ Yasushi Shimizu $^{2} \cdot$ Shin Kariya $^{3} \cdot$ Ryuji Yasumatsu $^{4} \cdot$ Tomoya Yokota $^{5} \cdot$ Takashi Fujii $^{6}$. \\ Kiyoaki Tsukahara ${ }^{7} \cdot$ Masafumi Yoshida $^{8} \cdot$ Kenji Hanyu $^{9}$. Tsutomu Ueda ${ }^{10}$ • Hitoshi Hirakawa ${ }^{11} \cdot$ Shunji Takahashi $^{12}$. \\ Takeharu Ono ${ }^{13}$. Daisuke Sano ${ }^{14}$. Moriyasu Yamauchi ${ }^{15} \cdot$ Akihito Watanabe $^{16} \cdot$ Koichi Omori $^{17}$. \\ Tomoko Yamazaki ${ }^{18} \cdot$ Nobuya Monden ${ }^{19} \cdot$ Naomi Kudo $^{20} \cdot$ Makoto Arai $^{21}$ • Daiju Sakurai ${ }^{22} \cdot$ Takahiro Asakage $^{23}$. \\ Issei Doi ${ }^{24} \cdot$ Takayuki Yamada $^{25}$ - Akihiro Homma ${ }^{26}$
}

Published online: 10 April 2021

(c) The Author(s) 2021

Correction to: International Journal of Clinical Oncology https://doi.org/10.1007/s10147-020-01829-0

The original article can be found online at https://doi.org/10.1007/ s10147-020-01829-0.

Akihiro Homma

ak-homma@med.hokudai.ac.jp

1 Department of Head and Neck Surgery, Aichi Cancer Center Hospital, Nagoya, Japan

2 Department of Medical Oncology, Faculty of Medicine and Graduate School of Medicine, Hokkaido University, Sapporo, Japan

3 Department of Otolaryngology, Head and Neck Surgery, Okayama University Hospital, Okayama, Japan

4 Department of Otolaryngology, Graduate School of Medical Sciences, Kyushu University, Fukuoka, Japan

5 Division of Gastrointestinal Oncology, Shizuoka Cancer Center, Nagaizumi, Japan

6 Department of Head and Neck Surgery, Osaka International Cancer Institute, Osaka, Japan

7 Department of Otorhinolaryngology, Head and Neck Surgery, Tokyo Medical University, Tokyo, Japan

8 Otorhinolaryngology and Head and Neck Surgery, The University of Tokyo Hospital, Tokyo, Japan

9 Head and Neck Oncology Center, International University of Health and Welfare, Mita Hospital, Tokyo, Japan

10 Department of Otorhinolaryngology, Head and Neck Surgery, Hiroshima University Hospital, Hiroshima, Japan

11 Department of Otorhinolaryngology, Head and Neck Surgery, University of the Ryukyu Hospital, Nishihara, Japan

12 Department of Medical Oncology, The Cancer Institute Hospital of Japanese Foundation for Cancer Research, Tokyo, Japan

13 Department of Otolaryngology, Head and Neck Surgery, Kurume University Hospital, Kurume, Japan

14 Department of Otolaryngology, Head and Neck Surgery, Yokohama City University Hospital, Yokohama, Japan

15 Department of Otolaryngology, Head and Neck Surgery, Saga University Hospital, Saga, Japan

16 Department of Otolaryngology, Head and Neck Surgery, Keiyukai Sapporo Hospital, Sapporo, Japan

17 Department of Otolaryngology, Head and Neck Surgery, Kyoto University Hospital, Kyoto, Japan

18 Division of Head and Neck Cancer Oncology, Miyagi Cancer Center, Sendai, Japan

19 Department of Head and Neck Surgery, National Hospital Organization Shikoku Cancer Center, Matsuyama, Japan

20 Department of Otorhinolaryngology, Hirosaki University Graduate School of Medicine, Hirosaki, Japan

21 Department of Medical Oncology, Chiba University Hospital, Chiba, Japan

22 Department of Otorhinolaryngology, Head and Neck Surgery, Chiba University Hospital, Chiba, Japan

23 Department of Head and Neck Surgery, Tokyo Medical and Dental University Medical Hospital, Tokyo, Japan

24 Medical Affairs, ONO Pharmaceutical Co., Ltd, Osaka, Japan

25 Japan Medical and Development, Bristol-Myers Squibb K.K., Tokyo, Japan

26 Department of Otolaryngology, Head and Neck Surgery, Faculty of Medicine and Graduate School of Medicine, Hokkaido University, Kita15 Nishi7, Kita-Ku, Sapporo, Hokkaido 060-8638, Japan 
The article Effectiveness and safety of nivolumab in patients with head and neck cancer in Japanese real-world clinical practice: a multicentre retrospective clinical study, written by Nobuhiro Hanai, Yasushi Shimizu, Shin Kariya, Ryuji Yasumatsu, Tomoya Yokota, Takashi Fujii, Kiyoaki Tsukahara, Masafumi Yoshida, Kenji Hanyu, Tsutomu Ueda, Hitoshi Hirakawa, Shunji Takahashi, Takeharu Ono, Daisuke Sano, Moriyasu Yamauchi, Akihito Watanabe, Koichi Omori, Tomoko Yamazaki, Nobuya Monden, Naomi Kudo, Makoto Arai, Daiju Sakurai, Takahiro Asakage, Issei Doi, Takayuki Yamada and Akihiro Homma, was originally published Online First without Open Access. With the author(s)' decision to opt for Open Choice the copyright of the article changed on January 18, 2021 to $\odot$ Author(s) 2021 and the article is forthwith distributed under a Creative Commons Attribution 4.0 International License, which permits use, sharing, adaptation, distribution and reproduction in any medium or format, as long as you give appropriate credit to the original author(s) and the source, provide a link to the Creative Commons licence, and indicate if changes were made. The images or other third party material in this article are included in the article's Creative Commons licence, unless indicated otherwise in a credit line to the material. If material is not included in the article's Creative Commons licence and your intended use is not permitted by statutory regulation or exceeds the permitted use, you will need to obtain permission directly from the copyright holder. To view a copy of this licence, visit http://creativecommons. org/licenses/by/4.0.

The Author also likes to revise the following in the Original publication as below:

In the section of "Incidence of $\mathrm{AE}$ "The median time to onset of any immune-related AEs was 8.7 (0.1 -43.7) weeks; (months should be weeks).

The original article has been corrected.

Open Access This article is licensed under a Creative Commons Attribution 4.0 International License, which permits use, sharing, adaptation, distribution and reproduction in any medium or format, as long as you give appropriate credit to the original author(s) and the source, provide a link to the Creative Commons licence, and indicate if changes were made. The images or other third party material in this article are included in the article's Creative Commons licence, unless indicated otherwise in a credit line to the material. If material is not included in the article's Creative Commons licence and your intended use is not permitted by statutory regulation or exceeds the permitted use, you will need to obtain permission directly from the copyright holder. To view a copy of this licence, visit http://creativecommons.org/licenses/by/4.0/.

Publisher's Note Springer Nature remains neutral with regard to jurisdictional claims in published maps and institutional affiliations. 